\title{
Hip 63510C, Hip 73786B, and nine new isolated high proper motion T dwarf candidates from UKIDSS DR6 and SDSS DR7
}

\begin{abstract}
R.-D. Scholz
Astrophysikalisches Institut Potsdam, An der Sternwarte 16, 14482 Potsdam, Germany

e-mail: rdscholz@aip.de

Received 16 February 2010 / Accepted 18 March 2010

ABSTRACT

Aims. Completing the poorly known substellar census of the solar neighbourhood, especially with respect to the coolest brown dwarfs, will lead to a better understanding of failed star formation processes and binary statistics with different environmental conditions. Methods. Using UKIDSS data and their cross-correlation with the SDSS, we searched for high proper motion mid- to late-T dwarf candidates with extremely blue near-infrared $(J-K<0)$ and very red optical-to-near-infrared $(z-J>+2.5)$ colours.

Results. With 11 newly found T dwarf candidates, the proper motions of which range between 100 and 800 mas/yr, we increased the number of UKIDSS $\mathrm{T}$ dwarf discoveries by $\approx 30 \%$. Large proper motions were also measured for six of eight previously known T4.5T9 dwarfs detected in our survey. All new candidates can be classified as T5-T9 dwarfs based on their colours. Two of these objects were found to be common proper motion companions of Hipparcos stars with accurate parallaxes. The latter allow us to determine absolute magnitudes from which we classify Hip 63510C as T7 and Hip 73786B as T6.5 dwarfs with an uncertainty of \pm 1 spectral subtype. The projected physical separation from their low-mass (M0.5 and K5) primaries is in both cases about 1200 AU. One of the Hipparcos stars has already a known very low-mass star or brown dwarf companion on a close astrometric orbit (Hip $63510 \mathrm{~B}=$ Gl 494B). With distances of only 11.7 and $18.6 \mathrm{pc}$, deduced from their primaries respectively for Hip 63510C and Hip 73786B, various follow-up observations can easily be carried out to study these cool brown dwarfs in more detail and to compare their properties with those of the already well-investigated primaries.
\end{abstract}

Key words. astrometry - proper motions - stars: distances - stars: kinematics and dynamics - brown dwarfs - solar neighborhood

\section{Introduction}

New deep near-infrared surveys like $\operatorname{UKIDSS}^{1}$ and the CanadaFrance Brown Dwarf Survey (CFBDS; Delorme et al. 2008b) provide a powerful tool for detecting even more of the coolest brown dwarfs (mid- and late-T dwarfs) in the solar neigbourhood than previously found in the SDSS (Abazajian et al. 2009) and 2MASS (Skrutskie et al. 2006). From 155 currently known T dwarfs (see Gelino et al. 2009 and references therein), there were 52 discovered with SDSS and 49 with 2MASS during the last 12 years. The number of discoveries from the UKIDSS large area survey (LAS) has already grown to 33 in only three years, where the majority were found by Lodieu et al. (2007) and Pinfield et al. (2008). The latest-type objects discovered in the SDSS and 2MASS are of spectral type T7 (Chiu et al. 2006) and T8 (Burgasser et al. 2002; Tinney et al. 2005; Looper et al. 2007) respectively, whereas a few T8.5-T9 dwarfs have been found in UKIDSS (Warren et al. 2007; Burningham et al. 2008, 2009) and CFBDS (Delorme et al. 2008a).

Since most of the stellar neighbours of the Sun were originally detected as high proper motion stars, one can expect to find the nearest cool brown dwarfs in new high proper motion

1 The UKIDSS project is defined in Lawrence et al. (2007). UKIDSS uses the UKIRT Wide Field Camera (WFCAM; Casali et al. 2007) and a photometric system described in Hewett et al. (2006) which is situated in the Mauna Kea Observatories (MKO) system (Tokunaga et al. 2002) The pipeline processing and science archive are described in Irwin et al. (in prep.) and Hambly et al. (2008). surveys using deep multi-epoch near-infrared imaging data over large areas of sky, as e.g. eventually provided by two epochs of $J$-band data in UKIDSS. However, one can already try to use the SDSS (Abazajian et al. 2009), the deepest available optical survey overlapping with the ongoing UKIDSS LAS, as the first epoch. The nearest cool brown dwarfs may still be visible in the red optical $z$-band of the SDSS data observed a few years before the UKIDSS observations started.

We describe a high proper motion survey for nearby T dwarfs using mainly UKIDSS and SDSS data which led to the discovery of 11 new mid- to late-T dwarf candidates, including two common proper motion objects of nearby red dwarf stars with accurate Hipparcos parallaxes.

\section{Selection of T dwarf candidates from UKIDSS}

For the selection of $\mathrm{T}$ dwarf candidates, we used the available cross-matching with the SDSS DR7 (Abazajian et al. 2009) as provided in the UKIDSS data base. To select faint objects that appear very red in optical-to-near-infrared colours, but blue in the near-infrared, as expected for mid- to late-T dwarfs, we used the following criteria:

$J 1>11, \quad J 1-K<0, \quad z-J 1>+2.5$,

where $J 1$ are the first epoch $J$-band data, and $z$ comes from the nearest matching SDSS object within the search radius of 10 arcsec. By doing so, we allowed for missing $Y$ - and $H$-band data. 
Table 1. SDSS DR7 $z$ and UKIDSS DR6 YJHK photometry for new T dwarf candidates.

\begin{tabular}{llllll}
\hline \hline Object & $z$ & $Y$ & $J$ & $H$ & $K$ \\
\hline ULAS J032920.22+043024.5 & $20.14 \pm 0.13^{*}$ & $18.67 \pm 0.05$ & $17.50 \pm 0.03$ & $17.88 \pm 0.07$ & $18.17 \pm 0.19$ \\
ULAS J081918.58+210310.8 & $20.73 \pm 0.21$ & $18.25 \pm 0.03$ & $16.95 \pm 0.01^{*}$ & $17.28 \pm 0.04$ & $17.18 \pm 0.06$ \\
ULAS J094516.39+075545.6 & $20.34 \pm 0.20$ & $18.71 \pm 0.03^{*}$ & $17.54 \pm 0.02^{*}$ & $17.76 \pm 0.04^{*}$ & $17.84 \pm 0.07^{*}$ \\
ULAS J101243.53+102101.6 & $20.22 \pm 0.20$ & $18.02 \pm 0.03$ & $16.88 \pm 0.01$ & $17.25 \pm 0.05$ & $17.45 \pm 0.08$ \\
ULAS J130041.72+122114.7 & $20.22 \pm 0.19$ & $17.72 \pm 0.03$ & $16.69 \pm 0.02$ & $17.01 \pm 0.04$ & $16.90 \pm 0.06$ \\
ULAS J141756.22+133045.8 & $20.42 \pm 0.17$ & $17.94 \pm 0.03$ & $16.77 \pm 0.01$ & $17.00 \pm 0.03$ & $17.00 \pm 0.04$ \\
ULAS J144901.90+114711.3 & $20.16 \pm 0.16$ & $18.35 \pm 0.04$ & $17.36 \pm 0.02$ & $17.73 \pm 0.07$ & $18.10 \pm 0.15$ \\
ULAS J150457.65+053800.8 & $19.88 \pm 0.13$ & $17.65 \pm 0.02$ & $16.59 \pm 0.02$ & $17.05 \pm 0.04$ & $17.41 \pm 0.09$ \\
ULAS J232035.28+144829.8 & $20.08 \pm 0.18$ & $17.94 \pm 0.03$ & $16.76 \pm 0.02$ & $17.11 \pm 0.04$ & $17.25 \pm 0.10$ \\
ULAS J232123.79+135454.8 & $20.11 \pm 0.17$ & $17.65 \pm 0.03$ & $16.69 \pm 0.02$ & $17.09 \pm 0.06$ & $17.36 \pm 0.10$ \\
ULAS J234228.96+085620.1 & $20.00 \pm 0.13$ & $17.42 \pm 0.02$ & $16.37 \pm 0.01$ & $16.73 \pm 0.03$ & $16.98 \pm 0.07$ \\
\hline
\end{tabular}

Notes. ${ }^{(*)}=$ mean values obtained from multiple measurements. ${ }^{(\dagger)}$ From two measurements $z=20.75 \pm 0.20$ and $z=19.53 \pm 0.18$, the latter is problematic (different from PSF magnitude). $z$ magnitudes are on the AB system. Y JHK magnitudes are aperMag3 derived for point sources (Dye et al. 2006) and are on the Vega system using the MKO photometric system.

Whereas our main search criterion was the negative $J-K$ colour, we excluded all kind of optically blue objects (early-type stars and white dwarfs) with the $z-J$ colour cut. Late-M and L dwarfs would also meet the $z-J$ colour but not the $J-K$ colour criterion. L subdwarfs (e.g. Lodieu et al. 2010) have negative $J-K$ but bluer $z-J(\approx+1.5)$. However, low-metallicity $\mathrm{T}$ dwarfs would possibly also satisfy our colour cuts, as the peculiar T6 dwarf 2MASS J0937+29 (Burgasser et al. 2006a) has $z-J \approx+3.5$ (derived from SDSS DR7 and Leggett et al. 2010 photometry). Note that a large $i-z$ was not required as a pre-condition. 4940 targets were found satisfying these criteria.

UKIDSS $Y, J 1, J 2, H$, and $K$ finding charts of all these candidates were inspected to exclude the vast majority of them consisting of ghost images of bright stars, images affected by diffraction spikes, satellite trails, galaxies, and asteroids. For the remaining star-like sources, the corresponding SDSS finding charts and DR7 data were checked for possible counterparts We have also used the SDSS database available at Princeton (Finkbeiner et al. 2004), where we found some additional epochs for determining the proper motions. Only 11 new candidates with a unique very red SDSS counterpart (detected only in the $z$-band with $z \approx 20$, whereas $i>23$ values listed in the SDSS DR7 indicate that there was no flux above the noise) within a smaller search radius of 5 arcsec and a significant proper motion were finally selected and are presented in Sect. 3. Note that these counterparts cannot be background late-M or L dwarfs, since they would show up in UKIDSS with $J \approx 18$ and $K \approx 16-17$, which is not the case. In addition, eight previously known T4.5T9 dwarfs were detected and proper motions determined for six of them with identified SDSS counterparts (Sect. 4).

We have also checked 2MASS, DENIS (Epchtein et al. 1997) and SSS (Hambly et al. 2001) I-band data for possible counterparts but failed to find any for the new $\mathrm{T}$ dwarf candidates due to their faintness.

\section{Astrometry and photometry of T dwarf candidates}

Full names and photometry of 11 new $\mathrm{T}$ dwarf candidates are listed in Table 1. All candidates have ugriz data where only the $z$ magnitudes are at or below the SDSS detection limit, whereas the ugri measurements are clearly above these limits. According
Table 2. Multi-epoch positions $\alpha, \delta$ (J2000.0) of common proper motion companions of Hipparcos stars.

\begin{tabular}{llllrl}
\hline \hline \multicolumn{3}{c}{ Object } & \multicolumn{5}{c}{ Epoch } & ID & Source \\
\multicolumn{7}{c}{${ }^{\prime}$} & \multicolumn{1}{c}{ yr } \\
\hline \multicolumn{7}{l}{ ULAS J1300+12 (Hip 63510C): } \\
41.9362 & 21 & 14.717 & 2003.223 & 3805 & SDSS DR7 \\
41.7711 & 21 & 14.754 & 2007.340 & 1465618 & UKIDSS $K$ \\
41.7709 & 21 & 14.747 & 2007.340 & 1467186 & UKIDSS $H$ \\
41.7286 & 21 & 14.695 & 2008.290 & 2321728 & UKIDSS $Y$ \\
41.7299 & 21 & 14.733 & 2008.290 & 2321748 & UKIDSS $J$ \\
\hline \multicolumn{7}{l}{ ULAS J1504+05 ((Hip 73786B): } \\
57.8614 & 38 & 03.278 & 2003.322 & 3910 & SDSS DR7 \\
57.6637 & 38 & 00.861 & 2008.151 & 1873794 & UKIDSS $H$ \\
57.6642 & 38 & 00.841 & 2008.151 & 1873842 & UKIDSS $K$ \\
57.6561 & 38 & 00.828 & 2008.293 & 2322073 & UKIDSS $Y$ \\
57.6561 & 38 & 00.849 & 2008.293 & 2322081 & UKIDSS $J$ \\
\hline
\end{tabular}

Notes. The ID is the run or multiframe number for SDSS or UKIDSS respectively.

Table 3. Common proper motions with Hipparcos stars in mas/yr.

\begin{tabular}{lccc}
\hline \hline Object & $\mu_{\alpha} \cos \delta$ & $\mu_{\delta}$ & $\varepsilon$ \\
\hline Hip 63510A & $-616.3 \pm 1.5$ & $-13.6 \pm 1.0$ & \\
ULAS J1300+12 & $-596 \pm 7$ & $+1 \pm 7$ & 23 \\
\hline Hip 73786A & $-608.8 \pm 2.7$ & $-502.7 \pm 3.6$ & \\
ULAS J1504+05 & $-614 \pm 4$ & $-496 \pm 8$ & 22 \\
\hline
\end{tabular}

Notes. Proper motions from linear fitting of the positions given in Table 2. For Hipparcos primaries data are taken from van Leeuwen (2007). $\varepsilon$ are the expected errors (see text).

to Abazajian et al. (2009), a 95\% detection repeatability for point sources is provided at $u=22.0, g=22.2, r=22.2, i=21.3$, $z=20.5$.

For the proper motion determination we took advantage of the sometimes slightly different epochs between the $Y J H K$-band measurements (up to one year epoch differences) and of the additional epochs (separated by several years from the UKIDSS epochs) provided by the SDSS (more details can be found in Tables 2 and 4). For the proper motion solutions we used linear fitting over all available epochs combined with a search for 
R.-D. Scholz: Hip 63510C, Hip 73786B, and nine new isolated high proper motion T dwarf candidates

Table 4. Multi-epoch positions $\alpha, \delta$ (J2000.0) of isolated objects.

\begin{tabular}{|c|c|c|c|c|c|}
\hline \multicolumn{3}{|c|}{ Object } & $\begin{array}{l}\text { Epoch } \\
\text { yr }\end{array}$ & ID & Source \\
\hline \multicolumn{6}{|c|}{ ULAS J0329+04: } \\
\hline 20.1535 & 30 & 24.390 & 2005.781 & 5714 & SDSS DR7 \\
\hline 20.1809 & 30 & 24.415 & 2006.816 & 6485 & SDSS DR7 \\
\hline 20.2211 & 30 & 24.558 & 2008.784 & 2336494 & UKIDSS $H$ \\
\hline 20.2227 & 30 & 24.538 & 2008.784 & 2336510 & UKIDSS $K$ \\
\hline 20.2170 & 30 & 24.549 & 2008.805 & 2338708 & UKIDSS $Y$ \\
\hline 20.2207 & 30 & 24.583 & 2008.805 & 2338724 & UKIDSS $J$ \\
\hline \multicolumn{6}{|c|}{ ULAS J0819+21: } \\
\hline 18.5794 & 03 & 11.246 & 2004.209 & 4508 & SDSS DR7 \\
\hline 18.5888 & 03 & 10.809 & 2007.049 & 1184392 & UKIDSS $J$ \\
\hline 18.5848 & 03 & 10.480 & 2008.907 & 2340123 & UKIDSS $H$ \\
\hline 18.5792 & 03 & 10.497 & 2008.907 & 2340143 & UKIDSS $K$ \\
\hline 18.5849 & 03 & 10.441 & 2008.910 & 2341325 & UKIDSS $Y$ \\
\hline 18.5809 & 03 & 10.430 & 2008.910 & 2341345 & UKIDSS $J$ \\
\hline \multicolumn{6}{|c|}{ ULAS J0945+07: } \\
\hline 16.1702 & 55 & 46.304 & 2002.194 & 3031 & SDSS DR7 \\
\hline 16.3889 & 55 & 45.586 & 2007.052 & 1186762 & UKIDSS $J$ \\
\hline 16.3974 & 55 & 45.632 & 2007.052 & 1188655 & UKIDSS $J$ \\
\hline 16.4008 & 55 & 45.652 & 2007.060 & 1343254 & UKIDSS $H$ \\
\hline 16.3905 & 55 & 45.650 & 2007.060 & 1343620 & UKIDSS $H$ \\
\hline 16.3977 & 55 & 45.636 & 2007.063 & 1330616 & UKIDSS $K$ \\
\hline 16.3937 & 55 & 45.589 & 2007.063 & 1332824 & UKIDSS $K$ \\
\hline 16.3947 & 55 & 45.584 & 2007.066 & 1335120 & UKIDSS $Y$ \\
\hline 16.3919 & 55 & 45.569 & 2007.066 & 1336848 & UKIDSS $Y$ \\
\hline \multicolumn{6}{|c|}{ ULAS J1012+10: } \\
\hline 43.6322 & 21 & 04.108 & 2002.953 & 3538 & SDSS DR7 \\
\hline 43.5471 & 21 & 01.826 & 2007.005 & 1147818 & UKIDSS $K$ \\
\hline 43.5350 & 21 & 01.683 & 2007.249 & 1354063 & UKIDSS $H$ \\
\hline 43.5350 & 21 & 01.698 & 2007.304 & 1415926 & UKIDSS $J$ \\
\hline 43.5356 & 21 & 01.698 & 2007.304 & 1417555 & UKIDSS $Y$ \\
\hline \multicolumn{6}{|c|}{ ULAS J1417+13: } \\
\hline 56.2478 & 30 & 45.565 & 2003.472 & 3996 & SDSS DR7 \\
\hline 56.2275 & 30 & 45.875 & 2007.367 & 1450483 & UKIDSS $Y$ \\
\hline 56.2263 & 30 & 45.868 & 2007.367 & 1450579 & UKIDSS $J$ \\
\hline 56.2226 & 30 & 45.943 & 2008.222 & 2318417 & UKIDSS $K$ \\
\hline 56.2236 & 30 & 45.915 & 2008.222 & 2318429 & UKIDSS $H$ \\
\hline \multicolumn{6}{|c|}{ ULAS J1449+11: } \\
\hline 01.9706 & 47 & 12.307 & 2003.409 & 3971 & SDSS DR7 \\
\hline 01.9050 & 47 & 11.390 & 2007.260 & 1367513 & UKIDSS $J$ \\
\hline 01.9064 & 47 & 11.415 & 2007.260 & 1368654 & UKIDSS $Y$ \\
\hline 01.9138 & 47 & 11.267 & 2007.293 & 1402270 & UKIDSS $K$ \\
\hline 01.9024 & 47 & 11.389 & 2007.293 & 1402702 & UKIDSS $H$ \\
\hline \multicolumn{6}{|c|}{ ULAS J2320+14: } \\
\hline 35.1020 & 48 & 28.996 & 2000.735 & 1739 & SDSS P \\
\hline 35.1064 & 48 & 28.978 & 2000.910 & 1904 & SDSS P \\
\hline 35.2839 & 48 & 29.835 & 2007.729 & 1716958 & UKIDSS $K$ \\
\hline 35.2900 & 48 & 29.815 & 2007.729 & 1716859 & UKIDSS $H$ \\
\hline 35.2914 & 48 & 29.815 & 2007.729 & 1717061 & UKIDSS $Y$ \\
\hline 35.2890 & 48 & 29.836 & 2007.729 & 1717217 & UKIDSS $J$ \\
\hline \multicolumn{6}{|c|}{ ULAS J2321+13: } \\
\hline 23.7591 & 54 & 59.058 & 2000.735 & 1739 & SDSS P \\
\hline 23.7724 & 54 & 58.799 & 2000.910 & 1904 & SDSS P \\
\hline 23.7910 & 54 & 58.261 & 2001.639 & 2507 & SDSS P \\
\hline 23.7990 & 54 & 54.895 & 2007.666 & 1689556 & UKIDSS $J$ \\
\hline 23.7990 & 54 & 54.943 & 2007.666 & 1690721 & UKIDSS $K$ \\
\hline 23.7990 & 54 & 54.918 & 2007.666 & 1690865 & UKIDSS $Y$ \\
\hline 23.7969 & 54 & 54.935 & 2007.666 & 1691223 & UKIDSS $H$ \\
\hline \multicolumn{6}{|c|}{ ULAS J2342+08: } \\
\hline 28.9358 & 56 & 20.123 & 2006.715 & 6354 & SDSS DR7 \\
\hline 28.9455 & 56 & 20.133 & 2007.901 & 1830486 & UKIDSS $H$ \\
\hline 28.9423 & 56 & 20.140 & 2007.901 & 1830585 & UKIDSS $K$ \\
\hline 28.9659 & 56 & 20.111 & 2008.732 & 2335155 & UKIDSS $Y$ \\
\hline 28.9655 & 56 & 20.105 & 2008.732 & 2335171 & UKIDSS $J$ \\
\hline
\end{tabular}

Notes. The ID is the run or multiframe number for SDSS or UKIDSS respectively. SDSS P stands for the SDSS data base in Princeton.
Table 5. Proper motions of isolated objects in mas/yr.

\begin{tabular}{lrrr}
\hline \hline Object & $\mu_{\alpha} \cos \delta$ & $\mu_{\delta}$ & $\varepsilon$ \\
\hline ULAS J0329+04 & $+323 \pm 15$ & $+60 \pm 07$ & 32 \\
ULAS J0819+21 & $+5 \pm 14$ & $-168 \pm 07$ & 24 \\
ULAS J0945+07 & $+685 \pm 13$ & $-142 \pm 07$ & 21 \\
ULAS J1012+10 & $-327 \pm 11$ & $-558 \pm 06$ & 25 \\
ULAS J1417+13 & $-76 \pm 03$ & $+77 \pm 03$ & 24 \\
ULAS J1449+11 & $-242 \pm 21$ & $-244 \pm 18$ & 27 \\
ULAS J2320+14 & $+387 \pm 05$ & $+121 \pm 02$ & 11 \\
ULAS J2321+13 & $+56 \pm 15$ & $-577 \pm 10$ & 10 \\
ULAS J2342+08 & $+229 \pm 55$ & $-9 \pm 09$ & 48 \\
\hline
\end{tabular}

Notes. Proper motions come from linear fitting of the positions given in Table 4. $\varepsilon$ are the expected errors (see text).

Table 6. Proper motions of known T dwarfs in mas/yr.

\begin{tabular}{llrrr}
\hline \hline Object & SpT (Ref) & $\mu_{\alpha} \cos \delta$ & $\mu_{\delta}$ & $\varepsilon$ \\
\hline SDSS J0325+04 & T5.5 (1) & $-185 \pm 06$ & $+15 \pm 07$ & 27 \\
2MASSI J0755+22 & T5.0 (2) & $-13 \pm 03$ & $-241 \pm 05$ & 11 \\
SDSS J0830+01 & T4.5 (2) & $+214 \pm 06$ & $-329 \pm 09$ & 08 \\
2MASS J1231+08 & T5.5 (2) & $-1185 \pm 09$ & $-1050 \pm 12$ & 14 \\
SDSS J1504+10 & T7.0(1) & $+338 \pm 13$ & $-350 \pm 11$ & 21 \\
2MASSI J2339+13 & T5.0 (2) & $+380 \pm 05$ & $-971 \pm 05$ & 12 \\
\hline
\end{tabular}

Notes. Proper motions as obtained from linear fitting of UKIDSS, SDSS, and (if available) 2MASS and DENIS positions. $\varepsilon$ are the expected errors (see text). For full names and references, see Gelino et al. (2009). Spectral type (SpT) references (Ref): (1) - Chiu et al. (2006); (2) - Burgasser et al. (2006b).

the correct counterparts at the expected positions in data from additional epochs.

The errors of the proper motion components given in Tables 3, 5, and 6 are estimated from the scatter around the bestfit line in each coordinate. and describe the quality of the fit. Due to the small number of available epochs, these formal errors may be unrealistically small (few data points may be well-aligned by chance) compared to the true errors. If we know the errors in the positions of the objects measured at different epochs in the SDSS and UKIDSS, we can estimate which error in their proper motions would be expected for the given epoch differences using error propagation.

The SDSS DR7 statistical errors per coordinate for bright stars are 45 mas, with systematic errors of less than 20 mas (Abazajian et al. 2009). However, for faint objects at the SDSS survey limit, the astrometric accuracy is limited by photon statistics to about 100 mas (Pier et al. 2003). The UKIDSS astrometric accuracy varies between 50 mas at low galactic latitudes and 100 mas at high galactic latitudes (Lawrence et al. 2007). For our objects, mostly located at intermediate galactic latitudes $\left(|b|=40^{\circ}-50^{\circ}\right)$, we assume uncertainties of 100 mas and 70 mas respectively for single SDSS and UKIDSS positions, which include smaller colour-dependent systematic errors in the astrometry expected to be of the order of a few tens of milliarcsec. We combine all available SDSS data of a given object in a mean first epoch and all UKIDSS data in a mean second epoch, where the accuracy of the mean positions is getting higher with the square root of the number of available single epochs, and compute the expected proper motion errors from error propagation. These errors $(\varepsilon)$ are given in the last column of Tables 3, 5, and 6. For 

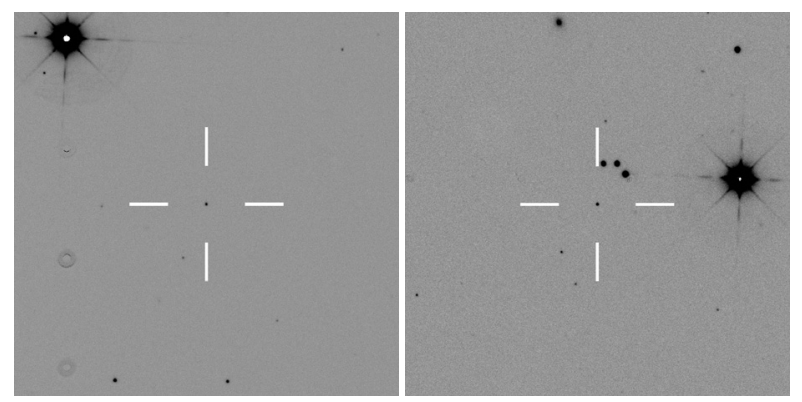

Fig. 1. $3 \times 3 \operatorname{arcmin}^{2}$ UKIDSS $J$-band images, left: ULAS J1300+12 (=Hip 63510C) with the bright primary, Hip 63510A in the upper left corner, right: ULAS J1504+05 (=Hip 73786B) with its primary, Hip 73786A on the right side. North is up, East to the left.

the previously known T dwarfs (Table 6), available 2MASS and DENIS positions were also assigned 100 mass individual errors and used together with the SDSS positions for the mean first epoch, which was then compared with the mean UKIDSS position to compute $\varepsilon$.

The expected proper motion errors $\varepsilon$ are on average two times larger than the formal proper motion errors. Nevertheless, all proper motions remain highly significant also compared to their $\varepsilon$ values, except for two objects with proper motions only 3-5 times larger than the expected errors (for further discussion, see Sect. 3.2). Note that the computed linear proper motions may be affected by parallactic motions for the nearest objects, since in most cases the different epoch observations were not made during the same time of the year (see Tables 2,4).

\subsection{Common proper motion companions of Hipparcos stars}

After each successful proper motion determination we checked if the newly found candidate is an already known $\mathrm{T}$ dwarf or if there are other known stars with similar large proper motions within a search radius of 2 degrees using SIMBAD. For two new candidates we succeeded to find Hipparcos stars with very similar proper motions (Table 3 ) based on which we consider the two T dwarf candidates as wide companions of these Hipparcos stars. ULAS J1300+12 (Hip 63510C) is separated by about 103 arcsec from Hip 63510A (Fig. 1, left panel) corresponding to a projected physical separation of $\approx 1200 \mathrm{AU}$ at $11.69 \pm 0.21 \mathrm{pc}$ distance as derived from the newly determined Hipparcos parallax of $85.54 \pm 1.53$ mas (van Leeuwen 2007). Hip 63510A (other names: Gl 494A; LHS 2665A), an active, rapidly rotating M0.5 dwarf (see e.g. Rauscher \& Marcy 2006; Browning et al. 2010), has already a known close brown dwarf candidate companion with an astrometric orbit of 14.5 years and resolved by adaptive optics (Gl 494B; Heintz 1994; Beuzit et al. 2004). For ULAS J1504+05 (Hip 73786B), the separation from its primary, the K5 dwarf (Cenarro et al. 2007) Hip 73786A (other names: Gl 576A; LHS 3020A), is about 68 arcsec (Fig. 1, right panel). This leads to a projected physical separation of $\approx 1260 \mathrm{AU}$ at $18.59 \pm 0.97 \mathrm{pc}$ distance obtained from the trigonometric parallax of $53.80 \pm 2.80$ mas (van Leeuwen 2007).

Goldman et al. (2010) have recently also reported on the discovery of Hip 63510C (=Ross 458C). Their proper motion measurement is based on the UKIDSS data and on additional new epoch observations with a total time baseline of two years leading to larger uncertainties compared to our proper motion using a five years baseline from SDSS and UKIDSS data.

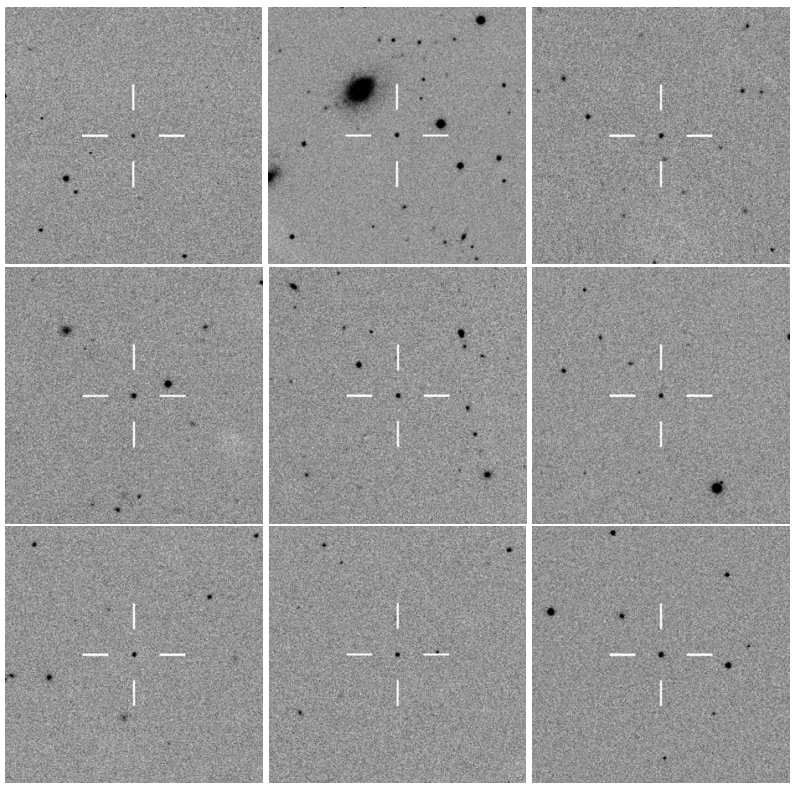

Fig. 2. $2 \times 2 \operatorname{arcmin}^{2}$ UKIDSS $J$-band images of ULAS J0329+04, ULAS J0819+21, ULAS J0945+07 (top row, from left to right), ULAS J1012+10, ULAS J1417+13, ULAS J1449+11 (middle row), ULAS J2320+14, ULAS J2321+13, and ULAS J2342+08 (bottom row). North is up, East to the left.

\subsection{Isolated T dwarf candidates}

The individual astrometric measurements of the remaining nine candidates, which appeared to have no common proper motion objects, are listed in Table 4. Their proper motions are given in Table 5. In most cases, there are more than just two effective epochs available, leading to a reliable linear proper motion fit. This is also true for the object with the smallest proper motion, ULAS J1417+13, which shows however the largest discrepancy between the very small formal proper motion errors and the expected proper motion error $\varepsilon$. But even compared to the latter, both proper motion components are three times larger, so significant. The reason for the relatively uncertain proper motion (4-5 times larger than its error) in case of ULAS J2342+08 is the late SDSS epoch and resulting short total time baseline (only 2 years).

\section{Proper motions of previously known T dwarfs}

In Table 6, the proper motions of six known $\mathrm{T}$ dwarfs from the compilation of Gelino et al. (2009) detected in our combined survey of UKIDSS and SDSS are listed. In part, they were determined using additional epoch data from 2MASS and DENIS.

For four of the known $\mathrm{T}$ dwarfs, these are the first proper motion measurements. For 2MASS J1231+08 and 2MASSI J2339+13, we have obtained similar but five to ten times more accurate proper motions than determined earlier by Burgasser et al. (2004) and Burgasser et al. (2003) respectively, even if we prefer the expected proper motion errors $\varepsilon$ to our formal errors.

\section{Conclusions and discussion}

Whereas for three of our candidates we measure moderate $z-J=$ $+2.6 \cdots+2.8$ meeting our selection criteria, eight objects have 
Table 7. Estimated spectral types of $\mathrm{T}$ dwarf candidates based on colours and absolute magnitudes.

\begin{tabular}{|c|c|c|c|c|c|}
\hline Object & $\operatorname{SpT}(J-H)$ & $\operatorname{SpT}(J-K)$ & $\operatorname{SpT}\left(M_{J}\right)$ & $\operatorname{SpT}\left(M_{K}\right)$ & SpT adopted \\
\hline ULAS J0329+04 & T5.0-T9.0 (-0.38) & T5.5-T9.0 (-0.67) & & & $\mathrm{T} 7.0 \pm 2.0$ \\
\hline ULAS J0819+21 & T5.0-T6.5 $(-0.33)$ & T4.0-T6.5 $(-0.23)$ & & & $\mathrm{T} 5.5 \pm 1.0$ \\
\hline ULAS J0945+07 & T4.5-T6.0 (-0.22) & T4.0-T7.5 $(-0.30)$ & & & $\mathrm{T} 5.5 \pm 1.5$ \\
\hline ULAS J1012+10 & T5.0-T9.0 (-0.37) & T5.5-T9.0 (-0.57) & & & $\mathrm{T} 7.0 \pm 2.0$ \\
\hline Hip 63510C & T5.0-T6.5 $(-0.33)$ & T4.0-T6.5 $(-0.22)$ & T7.5-T8.0 (16.35) & T6.5-T8.0 (16.56) & $\mathrm{T} 7.0 \pm 1.0$ \\
\hline ULAS J1417+13 & T5.0-T5.5 (-0.23) & T4.0-T6.5 $(-0.23)$ & & & $\mathrm{T} 5.5 \pm 1.0$ \\
\hline ULAS J1449+11 & T5.0-T9.0 (-0.37) & T5.5-T9.0 $(-0.75)$ & & & $\mathrm{T} 7.0 \pm 2.0$ \\
\hline Hip 73786B & T6.5-T9.0 (-0.46) & T6.0-T9.0 (-0.82) & T6.0-T7.5 (15.25) & T6.5-T7.5 (16.07) & $\mathrm{T} 6.5 \pm 1.0$ \\
\hline ULAS J2320+14 & T5.0-T9.0 (-0.34) & T5.0-T9.0 $(-0.49)$ & & & $\mathrm{T} 7.0 \pm 2.0$ \\
\hline ULAS J2321+13 & T5.5-T9.0 (-0.40) & T5.5-T9.0 $(-0.67)$ & & & $\mathrm{T} 7.5 \pm 1.5$ \\
\hline ULAS J2342+08 & T5.0-T9.0 $(-0.36)$ & T5.5-T9.0 $(-0.61)$ & & & $\mathrm{T} 7.0 \pm 2.0$ \\
\hline
\end{tabular}
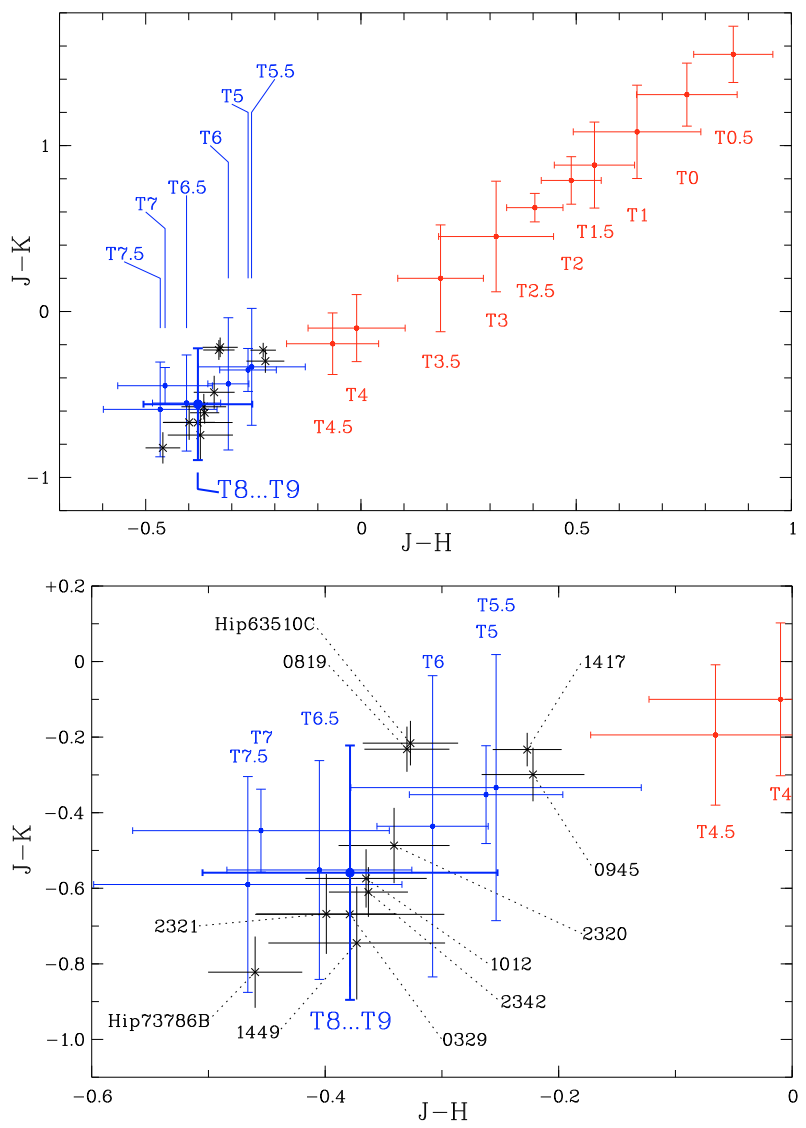

Fig. 3. Upper panel: Mean $J-K$ vs. $J-H$ (filled symbols) in MKO system for different sub-classes of $\mathrm{T}$ dwarfs (labelled; their error bars represent standard deviations) from Leggett et al. (2010) with the colours of our 11 candidates overplotted as crosses with error bars. Lower panel: Zoom to late $\mathrm{T}$ dwarf region, where the targets are also labelled.

much larger values of $z-J=+3.3 \cdots+3.8$ (ULAS J0819+21 has $z-J \approx+3.8$, ULAS J1417+13 and ULAS J2342+08 have $z-J \approx$ +3.6) approaching the typical values of T8-T9 dwarfs (Warren et al. 2007; Burningham et al. 2008, 2009; Delorme et al. 2008a).

The mean near-infrared colours $J-K$ and $J-H$ of known $\mathrm{T}$ dwarfs follow a clear trend to bluer colour with later subtype (Fig. 3), except for T8-T9 dwarfs which again show colours similar to T6-T7.5 dwarfs. All our new T dwarf candidates exhibit sufficiently blue colours classifying them as $\geq \mathrm{T} 5$ dwarfs. Based on these colours we assign a preliminary classification as $\approx \mathrm{T} 5.5$ (with uncertainties up to \pm 1.5 subtypes) for ULAS J0819+21, ULAS J0945+07, ULAS J1300+12 (Hip 63510C), and ULAS $\mathrm{J} 1417+13$ (both $J-K$ and $J-H$ are between -0.2 and -0.35 ). For the remaining objects with even larger negative colour indices, including ULAS J1504+05 (=Hip 73786B) with the most extreme values $J-K \approx-0.8$ and $J-H \approx-0.5$, the colours indicate T5-T9 spectral types (Table 7). The larger uncertainty comes here mainly from the already mentioned colour reversal of T8-T9 dwarfs.

Absolute magnitudes of the two Hipparcos star companions can be derived using the accurate distances of their primaries. Taking into account the errors in the distances and apparent magnitudes, we get $M_{J}$ of $16.35 \pm 0.04$ and $15.25 \pm 0.12$, and $M_{K}$ of $16.56 \pm 0.07$ and $16.07 \pm 0.15$ respectively for Hip $63510 \mathrm{C}$ and Hip 73786B. These absolute magnitudes place Hip 63510C among the T6-T8 dwarfs with trigonometric parallaxes as given in Leggett et al. (2010), whereas Hip 73786B is as faint as T5.5T7.5 dwarfs listed in that paper. The fact that the colours hint at a later spectral type for Hip 73786B compared to Hip 63510C, whereas the classification by absolute magnitudes preferred by us lead to a different conclusion, underlines the need for spectroscopic observations of our new targets and direct comparison with template spectra of the coolest known brown dwarfs. Goldman et al. (2010) discuss Hip 63510C in more detail and come to the conclusion that it is a T8-T9 dwarf with an absolute magnitude and $J-K$ colour pointing to a young age or possible binarity.

Acknowledgements. Data from the UKIDSS 6th data release, SDSS DR7, 2MASS, and DENIS served as the basis for this work. We have also used the SIMBAD and VizieR services at the CDS in Strasbourg and the ARICNS data base on nearby stars in Heidelberg. We thank Doug Finkbeiner for his help accessing the SDSS data at Princeton University and Axel Schwope for comments on a first version of the manuscript. We also thank the referee, Dr. P. Delorme, for helpful comments which led to an improved and extended paper.

\section{References}

Abazajian, K. N., Adelman-McCarthy, J. K., Agüeros, M. A., et al. 2009, ApJS, 182,54

Beuzit, J.-L., Segransan, D., Forveille, T., et al. 2004, A\&A, 425, 997

Browning, M. K., Basri, G., Marcy, G. W., West, A. A., \& Zhang, J. 2010, AJ, 139, 504

Burgasser, A. J., Kirkpatrick, J. D., Brown, M. E., et al. 2002, ApJ, 564, 421 Burgasser, A. J., Kirkpatrick, J. D., McElwain, M. W., et al. 2003, AJ, 125, 850 Burgasser, A. J., McElwain, M. W., Kirkpatrick, J. D., et al. 2004, AJ, 127, 2856 Burgasser, A. J., Burrows, A., \& Kirkpatrick, J. D. 2006a, ApJ, 639, 1095

Burgasser, A. J., Geballe, T. R., Leggett, S. K., Kirkpatrick, J. D., \& Golimowski, D. A. 2006 b, ApJ, 637, 1067 
Burningham, B., Pinfield, D. J., Leggett, S. K., et al. 2008, MNRAS, 391, 320 Burningham, B., Pinfield, D. J., Leggett, S. K., et al. 2009, MNRAS, 395, 1237

Casali, M., Adamson, A., Alves de Oliveira, C., et al. 2007, A\&A, 467, 777

Cenarro, A. J., Peletier, R. F., Sánchez-Blázquez, P., et al. 2007, MNRAS, 374, 664

Chiu, K., Fan, X., Leggett, S. K., et al. 2006, AJ, 131, 2722

Delorme, P., Delfosse, X., Albert, L., et al. 2008a, A\&A, 482, 961

Delorme, P., Willott, C. J., Forveille, T., et al. 2008b, A\&A, 484, 469

Dye, S., Warren, S. J., Hambly, N. C., et al. 2006, MNRAS, 372, 1227

Epchtein, N., de Batz, B., Capoani, L., et al. 1997, The Messenger, 87, 27

Finkbeiner, D. P., Padmanabhan, N., Schlegel, D. J., et al. 2004, AJ, 128, 2577

Gelino, C. R., Kirkpatrick, J. D., \& Burgasser, A. J. 2009, online database for $752 \mathrm{~L}$ and $\mathrm{T}$ dwarfs at DwarfArchives.org, status: 16 November

Goldman, B., Marsat, S., Henning, T., Clemens, C., \& Greiner, J. 2010, accepted by MNRAS, [arXiv: 1002.2637]

Hambly, N. C., MacGillivray, H. T., Read M. A., et al. 2001, MNRAS, 326, 1279

Hambly, N. C., Collins, R. S., Cross, N. J. G., et al. 2008, MNRAS, 384, 637
Heintz, W. D. 1994, AJ, 108, 2338

Hewett, P. C., Warren, S. J., Leggett, S. K., \& Hodgkin, S. T. 2006, MNRAS, 367,454

Lawrence, A., Warren, S. J., Almaini, O., et al. 2007, MNRAS, 379, 1599

Leggett, S. K., Burningham, B., Saumon, D., et al. 2010, ApJ, 710, 1627

Lodieu, N., Pinfield, D. J., Leggett, S. K., et al. 2007, MNRAS, 379, 1423

Lodieu, N., Zapatero Osorio, M. R., Martín, E. L., Solano, E., \& Aberasturi, M. 2010, ApJ, 708, L107

Looper, D. L., Kirkpatrick, J. D., \& Burgasser, A. J. 2007, AJ, 134, 1162

Pier, J. R., Munn, J. A., Hindsley, R. B., et al. 2003, AJ, 125, 1559

Pinfield, D. J., Burningham, B., Tamura, M, et al. 2008, MNRAS, 390, 304

Rauscher, E., \& Marcy, G. W. 2006, PASP, 118, 617

Skrutskie, M. F., Cutri, R. M., Stiening, R., et al. 2006, AJ, 131, 1163

Tinney, C. G., Burgasser, A. J., Kirkpatrick, J. D., \& McElwain, M. W. 2005, AJ, 130,2326

Tokunaga, A. T., Simons, D. A., \& Vacca, W. D. 2002, PASP, 114, 180

van Leeuwen, F. 2007, A\&A, 474, 653

Warren, S. J., Mortlock, D. J., Leggett, S. K., et al. 2007, MNRAS, 381, 1400 